East African Medical Journal Vol. 77 No. 10 October 2000

MALNUTRITION: MORE THAN THE EYE CAN SEE

F.C.M. van Leth, MD, DTM\&H, Medical Officer in-Charge, J.M. Koeleman, MD, DTM\&H, Medical Officer and A.S. Manya, MBBS, Medical Officer, Misikhu Mission Hospital, P.O. Box 129 Webuye, Kenya

Request for reprints to: Dr. F.C.M. van Leth, William Temple House, 29, Trebovir Road, London SW5 9NQ, United Kingdom

\title{
MALNUTRITION: MORE THAN THE EYE CAN SEE
}

\author{
F.C.M. van LETH, J.M. KOELEMAN and A.S. MANYA
}

\begin{abstract}
Objectives: To assess the magnitude of malnutrition in a hospital setting and to relate anthropometric measures to the clinical diagnosis of malnutrition.

Design: A descriptive study whereby anthropometric measures (length and weight) were taken of every child under the age of five years who was admitted to the hospital. The anthropometric data were analysed using the EPI-Info statistical package, which calculates z-scores for weight-for-age, weight-for-height and height-for-age. As reference curve, the reference growth curves of the NCHS were used. Of all the children who were classified as being malnourished, it was recorded if the clinical diagnosis of malnutrition was made at the time of admission or during the hospital stay.

Setting: Misikhu Mission Hospital, western Kenya.

Subjects: Every child under the age of five years who was admitted to the hospital, was eligible to enter the study. The data of 1130 children were used. The data of 40 other children who were admitted in this period were not complete and could therefore not be used.

Results: An overall percentage for malnutrition of $\mathbf{4 4 . 3}$ was found. Only fourteen per cent of the malnourished children were clinically diagnosed as having malnutrition.

Conclusion: Anthropometric measures are an easy, but time-consuming way of identifying children with malnutrition, it identifies more children with malnutrition than clinical diagnosis alone. Therefore it should be considered to implement standardised anthropometry in a hospital setting.
\end{abstract}

\section{INTRODUCTION}

Malnutrition is still a wide-spread problem in Kenya. According to UNICEF an overall percentage for wasting of eight per cent is found among children below the age of five years. Thirty four per cent of the children in this age group are stunted(1).

Misikhu Mission Hospital is a moderately large mission hospital with 171 beds in rural western Kenya. Yearly between 6000 and 8000 patients are admitted of which a large part (35-45\%) consists of children below the age of five years. Due to lack of resources, the diagnosis of malnutrition is made only on clinical aspects. The only other tool available in Misikhu Mission Hospital, and many other hospitals in western Kenya, is anthropometry. This is not a standard procedure on admission because it is regarded time-consuming. To assess the problem of malnutrition in a hospital setting, as well as to relate clinical diagnosis to anthropometric measurements, a descriptive study was carried out. This report describes the findings and discusses possible implications.

\section{MATERIALS AND METHODS}

During a four month period (May-August 1997), every child below the age of 60 months who was admitted to the children's ward of the hospital was eligible to enter the study. On admission the weight $(\mathrm{kg})$ and the length $(\mathrm{cm})$ were established. Children below the age of 12 months were weighed using a Salter hanging scale up to 100 grams accurate. The scales were adjusted every day. The older children were weighed using a standing weighing scale with the same accuracy. The height was measured lying down in children below the age of 12 months, and standing up in children above that age. All parameters were measured and recorded by one of the three Medical Officers conducting the study. At the end of the study period, the data were analysed with the EPI INFO software package (version 6.0). EPI-INFO calculates anthropometric indices as weight-for-age, height-forage and weight-for-height, using the growth reference curves developed by the National Center for Health Statistics (NCHS). The use of these reference curves is recommended by the $\mathrm{WHO}(2)$. The indices are calculated as Z-scores. A total of 1170 children were eligible to enter the study. The parameters of 40 children were not used because they were incomplete. The definitions used for wasting and stunting are those recommended by the WHO, in which wasting is defined as 'weight for height' $\leq 2 \mathrm{SD}$ from the mean, and stunting is defined as 'height for age' $\leq 2$ SD from the mean.

\section{RESULTS}

Table 1 shows the parameters of the children in the study population. Out of the 1130 children examined 501 $(44.3 \%)$ were wasted, stunted or both. The classification of wasted, stunted or both is exclusive which means that no child is entered more than once in the calculations. 
Despite this high figure, the clinical diagnosis of malnutrition was only made at discharge for $14.1 \%$ of the wasted children, $11.2 \%$ of the stunted children and $42.2 \%$ of the children who were both wasted and stunted.

Table 1

Parameters of study group $(n=1130)$

\begin{tabular}{|c|c|c|c|}
\hline & Male & Female & $\mathrm{p}$-value \\
\hline Number (\%) & $582(51.5)$ & $548(48.5)$ & 0.31 \\
\hline $\begin{array}{l}\text { Mean age (SD) } \\
\text { (months }\end{array}$ & $18.0(11.5)$ & $17.7(11.9)$ & 0.66 \\
\hline Mean WAZ (SD) & $-1.9(1.7)$ & $-1.9(1.4)$ & \\
\hline Mean WHZ (SD) & $-1.4(1.3)$ & $-1.3(1.3)$ & 0.19 \\
\hline Mean HAZ (SD) & $-1.3(1.7)$ & $-1.3(1.4)$ & \\
\hline Wasted (\%) & $99(17.0)$ & $86(15.7)$ & 0.55 \\
\hline Dead (\%) & $12(12.1)$ & $11(12.9)$ & 0.64 \\
\hline Stunted (\%) & $88(15.1)$ & $92(16.8)$ & 0.44 \\
\hline Dead (\%) & $10(11.4)$ & $11(12.1)$ & 0.71 \\
\hline Wasted/stunted (\%) & $81(13.9)$ & $55(10.0)$ & 0.05 \\
\hline Dead (\%) & $17(21.3)$ & $21(38.2)$ & $<0.001$ \\
\hline
\end{tabular}

$\mathrm{WAZ}=\mathrm{z}$-score weight for age, $\mathrm{WHZ}=\mathrm{z}$-score weight for height; HAZ $=\mathrm{z}$-score height for age

The negative values for the means of the anthropometric indices indicate that the study population has a poor nutritional status as compared to the reference population used. The proportion of children being only wasted or only stunted did not differ for boys or girls, but the group of children who are both wasted and stunted comprised of significantly more boys than girls $(\mathrm{p}=0.05)$. In this group the death rate is significantly higher for girls than for boys $(\mathrm{p}<0.001)$.

\section{DISCUSSION}

It is generally accepted that wasting is an indicator for acute malnutrition, whereas stunting is an indicator for chronic malnutrition resulting from an inadequate intake of food for a relatively long period of time and frequent episodes of infection(3). In a hospital setting like Misikhu Mission Hospital, only the problem of wasting can be addressed since the many socio-economic factors leading to stunting can not be influenced at an institutional level.

The most striking outcome of the study is the acknowledgement of the poor match between anthropometric indices and the clinical diagnosis of malnutrition. This has far reaching consequences, since specific therapy is only started when the diagnosis of malnutrition is made on or during admission. In Misikhu Mission Hospital, this therapy consists of deworming the child, treatment of (seen and unseen) infections, supplementation of vitamins and minerals and the administration of a special diet (UNIMIX). When it is not recognised that a child is malnourished, this total package of treatment is not given, denying the child proper treatment.
It became clear that the clinical diagnosis of malnutrition is kept for children who are obviously marasmic or are suffering from kwashiorkor. All other presentations of malnutrition are easily overlooked. It might be that the sight of a child who is merely underweight or of short stature is such a common feature in the hospital that this condition does not raise any suspicion with the Medical Officers. The parents of these children do not see a reason to attend the hospital for malnutrition unless the child is clearly marasmic or kwashiorkor. The poor match between anthropometric indices and clinical diagnosis, together with the difference in appraisal of the nutritional status of the child by the parents and the anthropometric indices might be less striking when specific reference curves are used for African children.

The mean anthropometric indices are low, indicating a poor nutritional status of the children in the study population. This might have several reasons. Being a descriptive study in a hospital, the study population tends to be biased because all the children are sick. The majority of children (60\%) were seen for an acute illness (malaria, anaemia, pneumonia) and not for nutritional problems. This might be an argument that the study population does reflect the situation outside the hospital, for if the acute illness did not have arisen, the children would still be in the community representing a non-hospital population. This argument is stronger for the stunted children and for the children who are both wasted and stunted, because their nutritional situation has developed over a longer period of time. Another reason for the low mean anthropometric indices can be the fact that a reference curve is used which is developed for Caucasian children. The discussion whether or not specific reference curves need to be developed and used for African children is not new. Walraven et al (4) point out that reference curves for birthweight are susceptible to differences among different ethnicities. If these differences exists at birth, it is possible that the differences become more extreme during the first five years of life, resulting in low mean values for anthropometric indices when compared to the NCHS reference curves.

Two significant differences were found in the study, which were not expected. In the group of children who are both wasted and stunted, more boys were present $(\mathrm{p}=0.05)$. There was no sex difference in the other groups. No clear explanation can be given for this finding. It is known that in the catchment area of the hospital, boys are regarded superior to girls. When a boy falls severely ill, the parents might go to a greater length to find treatment for him. A girl in the same situation might be left home to die. Why the girls in this group had a higher death rate $(\mathrm{p}<0.001)$ is also not clear, because the mean nutritional status for boys and girls did not differ in this group as reflected in the mean values of the anthropometric indices. The "WAZ" was -4.2 and -4.0 for boys and girls respectively. The "WHZ" was -3.0 and -2.9 respectively and the "HAZ" was -3.5 and -3.2 respectively. 
More children were diagnosed as having malnutrition when anthropometry was carried out than when the diagnosis was made on clinical judgement alone. However, it should be stressed that malnutrition is not something defined by anthropometry, but rather a condition which indicates significant risk of pathology, morbidity and mortality. Therefore, anthropometry must be seen as a tool in diagnosing malnutrition and not as a golden standard.

In conclusion, as a result of the study, the problem of malnutrition in Misikhu Mission Hospital became more clear. Ways have to be found to improve diagnosing malnutrition as it tends to be overlooked when only done clinically. With simple but time-consuming anthropometric measurements, more children with malnutrition can be identified and subsequently treated. In many hospitals these anthropometric measurements are routinely carried out only at the antenatal clinics. This descriptive study shows that anthropometric measurements are also of value in an in-patient setting since it identifies children with malnutrition that are easily overlooked with clinical judgement alone.

\section{ACKNOWLEDGEMENTS}

To Dr. P. Zwanikken, and Dr. M.A. Brouwer, for their contributions to the text of this article.

\section{REFERENCES}

1. The state of the World's children 1998. UNICEF.

2. WHO working group. Use and interpretation of anthropometric indicators of nutritional status. WHO Bull 1986; 64: 929-41.

3. E. Tunçbilek, T Unalan and T. Coskun. Indicators of nutritional status in Turkish pre-school children: results of Turkish Demographic and Health survey. J. trop. Paediat. 1996; 42:78-84.

4. G.E.L. Walraven, R.J.B. Mkanje, H.A.G.H. van Asten, J van Roosmalen, P.W.J. van Dongen, W.M.V. Dolmans. The aetiology of low birthweight in a rural area of Tanzania. Trop. Med. Hlth. 1997; 2:558-67. 